\title{
HEMA/MMA/EDMA Packing Material Evaluation for Size Exclusion Chromatography (SEC)
}

\author{
Cristina Duarte Vianna-Soares ${ }^{\mathrm{a} *}$, Cherng-Ju Kim ${ }^{\mathrm{b}}$, Michael Robert Borenstein $^{\mathrm{c}}$ \\ ${ }^{a}$ Universidade Federal de Minas Gerais, Faculdade de Farmácia, \\ 31270-901 Belo Horizonte - MG, Brazil \\ '92354-2741, Loma Linda University, School of Pharmacy, Loma Linda, CA, 92354-274 \\ ${ }^{\mathrm{c}}$ Temple University School of Pharmacy, Department of Pharmaceutical Sciences, \\ Philadelphia, PA, 19140, United States
}

Received: September 17, 2003; Revised: October 9, 2004

\begin{abstract}
HEMA/MMA/EDMA (2-hydroxyethyl methacrylate/methyl methacrylate/ethyleneglycol methacrylate) copolymer beads have been synthesized for use in aqueous SEC. This porous chromatographic support consisted of a large average particle size $(250 \mu \mathrm{m})$ and contained macropores with a median pore size of approximately $880 \AA$ A. The material was packed in glass and stainless columns to assess their chromatographic performance. The packed columns were calibrated using narrow molecular weight (MW) distribution standards (dextran) and exhibited a range of molecular weight separation between 40,000 and 2,000,000 daltons. The packing material showed the ability to separate large molecules through the size exclusion mechanism.
\end{abstract}

Keywords: hydrophilic acrylate packing materials, size exclusion chromatography, macromolecules separation

\section{Introduction}

Since synthetic macroporous beads made of rigid organic materials such as polystyrene cross-linked with divinylbenzene were introduced by Moore ${ }^{1}$ in 1964, they have become widely employed in SEC due to their mechanical strength and chemical stability ${ }^{2}$. However, they possess hydrophobic characteristics that hinder their use in aqueous medium for hydrophilic SEC.

Ideally, SEC requires a hydrophilic support, which can be a silica material or a synthetic polymer. Silica-based packing materials are not as chemically stable as polymers gels, and polymer gels are not as efficient as silica ${ }^{3}$. Most synthetic packings based on hydrophilic materials are obtained by linking the functionalities directly onto the core of the beads or via a short spacer.

There have been numerous attempts at overcoming multiple synthetic steps, improving polymer efficiency and designing new porous material by using different polymerization methods, monomer compositions, solvents, initiators, etc ${ }^{4}$. The copolymerization of HEMA/MMA/ EDMA using different porogen (dodecanol, DOD) concentrations has been described in an effort to obtain hydrophilic packing materials the synthesis of porous beads. These polymer particles revealed great porosity, as well as, chemical and mechanical resistance.

Following the manufacture of a sorbent material, it must be packed into a column and characterized through a calibration curve with narrow molecular weight reference standards. Calibration is performed for the relative SEC methodology so that the statistical average molecular weights ( $\mathrm{Mw}=$ weight average molecular weight; $\mathrm{Mn}=$ number average molecular weight) and the MW distribution of the macromolecule sample can be determined. Absolute measurements without the need of a calibration are possible with the use of either an on-line light scattering detector or an on-line viscometer with universal calibration 5 . Pure and narrow MW dispersity (Mw/ $\mathrm{Mn} \cong 1$ ) are used as SEC calibration standards. Common macromolecules utilized for calibration purposes are for example polystyrene (non-aqueous SEC), polyethylene glycol (PEG), polyethylene oxide (PEO), polyacrylamide and dextrans (aqueous SEC). The calibration equation, generally defined by $Y=-b X+a$, describes the curve. The dependent variable, column retention volume, is represented in the $\mathrm{X}$ axis and the independent variable, standard MW, is represented in the $\mathrm{Y}$ axis for better visualization. The linear portion of the curve is the effective separation range of the SEC columns. High column selectivity is determined by the columns' narrow pore size distribution, high degree of homogeneity and the low slope of the calibration curve ${ }^{6}$. Calibration graphs with a small slope (shallow curve) will indicate high selectivity but in a rather limited working range, i.e., the column will resolve molecules of similar size. Steeper and larger calibration slopes are less selective, however they reach a wider range of MW separation ${ }^{7}$. In addition, deviations from linearity can occur and be indicative of non-specific interactions, unusual shapes of molecule or multimodal pore size distribution.

This work reports the chromatographic performance of beads prepared with 20\% DOD and 10\% EDMA as described earlier ${ }^{8}$. The beads were produced using the molar ratio of monomers (HEMA: MMA) to cross-linker (EDMA) of 1:1.30:0.19. The Scanning Electron Microscopy (SEM) characterized particles presented a median pore size of $880 \AA$ and a total pore volume of $74 \times 10^{-3} \mathrm{~mL} / \mathrm{g}$ measured by Mercury Intrusion Porosimetry (MIP).

\section{Materials and Methods}

\subsection{HEMA/MMA/EDMA beads preparation}

The synthesis and characterization of the HEMA/MMA/EDMA beads (2-hydroxyethyl methacrylate/methyl methacrylate/ethyleneglycol methacrylate copolymer) have been described elsewhere ${ }^{8}$. Briefly, a free radical suspension polymerization in the presence of an aqueous medium containing the inorganic gel medium, magnesium hydroxide, prepared in situ resulted in spherical particles. Dodecanol (DOD) was used as a porogen solvent. The polymerization was carried out at $70^{\circ} \mathrm{C}$ and an agitation rate of $250 \mathrm{rpm}$ in the presence of 2 , 2, azobis-2-methylpropionitrile (AIBN) as initiator. The beads were 
collected via vacuum filtration through fiberglass filter $(9 \mathrm{~mm})$ and dried in the vacuum oven overnight $\left(75 \mathrm{~cm} \mathrm{Hg}, 60^{\circ} \mathrm{C}\right)$. Simple extraction (SE, $3 \times 400 \mathrm{~mL}, 30$ minutes, magnetic agitation) and Soxhlet extraction (SOX, $400 \mathrm{~mL}, 17$ to 24 hours, $65^{\circ} \mathrm{C}$ ) of HEMA/MMA/ EDMA beads were proceeded to eliminate organics and prepare for aqueous packing. Soxhlet extraction was carried for all batches of beads previously extracted by simple extraction.

\subsection{SEC using the packed HEMA/MMA/EDMA material}

\subsubsection{HEMA/MMA/EDMA beads packing}

The beads were suspended in water and acetonitrile and the material was sedimented several times to eliminate fine particles. The SEC columns were prepared using a concentrated aqueous slurry suspension $(32.5 \% \mathrm{w} / \mathrm{v})$ of the beads and packed in a glass column $(50 \times 1.5 \mathrm{~cm}$ i.d.) under low pressure using a constant velocity peristaltic pump (Cole Parmer, Vernon Hills, PA). A DSF122 reciprocating plunger pump (Haskel Engineering, Burbank, CA) was also employed to pack a stainless steel column $(300 \times 4.6 \mathrm{~mm}$ i.d. $)$ using medium pressure (1345 psi). At least 5 column volumes were collected for each column during the packing process. SEM pictures of the post-packed particles were also evaluated.

\subsubsection{Calibration of HEMA/MMA/EDMA packed columns}

A series of dextran standards, in this work, designated as dextran standards A (MW 2,000,000, MW 600,000, MW 170,000, MW 75,000, MW 40,000 and MW 10,000), obtained from Polysciences, Inc., (Warrington, PA) was used to calibrate the SEC columns. Non-standards Dextran series, available from Aldrich Chemical Co. Inc. (Milwaukee, WI), were designated as B (MW 5-40,000,000; 2,000,000, MW 473,000, MW 76,000, MW 43,000 and MW0 $10,000)$. Each dextran series was injected in duplicate and the average of the elution volume peak was reported. Polysaccharides samples, Xanthan Gum/XAG (MW > 1,000,000), Sodium Alginic Acid/SAG (MW 120,000-190,000), Agarose/AGA (MW not defined), and proteins samples, Bovine Serum Albumin/BSA (MW 66,000), Ovalbumin/OVA (MW 44,000) and Cytochrome C/CYT (MW 12,375) obtained from Sigma Chemicals (St. Louis, MO) were used to test the calibrated columns. Aqueous mobile phases were $0.1 \% \mathrm{w} / \mathrm{v}$ sodium azide in water for the polysaccharides, and $0.01 \mathrm{M}$ phosphate buffer solution (PBS) pH 6.5 and $0.01 \mathrm{M}$ TRIS buffer $\mathrm{pH} 8.0$ containing $0.02 \mathrm{M}$ or $0.2 \mathrm{M}$ sodium sulfate for the proteins, at $1 \mathrm{~mL} / \mathrm{min}$ or $0.3 \mathrm{~mL} / \mathrm{min}$ flow rate with refractive index (RI) detection. Glucose was used as the column marker for the HEMA/MMA/EDMA packing material to evaluate the mobile phase volume $(\mathrm{Vm})$.

All chemicals were used without further purification.

\subsection{Methods}

A Spectra-Physics Liquid Chromatograph P100 (Thermo Separation Products, San Jose, CA) with a manual injection loop valve of 20 or $100 \mu \mathrm{L}$ and isocratic mobile phase elution at room temperature was employed. Detection was accomplished with either an RID-6A Refractive Index Detector (Shimadzu Corp., Kyoto, Japan) or a UV-100 Spectra-Physics ultraviolet detector (UV) connected in series and attached to a Recordall Series 5000 register (Fisher Scientific, Fair Lawn, $\mathrm{NJ}$ ) with $0.25 \mathrm{~cm} / \mathrm{min}$ chart speed $(s)$ and 10 or $100 \mathrm{mV}$ attenuation. The packed columns were initially equilibrated with deionized, distilled water at an adjusted flow rate $(f)$ and prior to use in SEC.

\section{Results and Discussion}

The chromatographic assays were conducted with the HEMA MMA:EDMA copolymer beads (molar ratio 1:1.30:0.19) prepared using 20\% w/w dodecanol as the porogen. During their preparation, it was observed that the solvent extraction procedure on the beads played an important role in the exposure and efficiency of the pores. It is worth noting that the efficiency of Soxhlet method used to extract the porogen and synthesis by-products from the beads was superior when compared to a simple extraction alone $e^{8}$. Therefore, in the absence of undesirable organic substances the beads could be adequately dried and the material prepared for use in SEC. Figures 1a and $1 \mathrm{~b}$ show the SEM pictures of the simple extraction and the Soxhlet extraction of HEMA/MMA/EDMA beads prepared with $20 \%$ dodecanol, respectively. The particles size approximated $250 \mu \mathrm{m}$. The median pore size and total pore volume were $880 \AA$ and $74 \times 10^{-3} \mathrm{~mL} / \mathrm{g}$, respectively, as indicated by Mercury Intrusion Porosimetry (MIP) analyses ${ }^{9,10}$.

SEM pictures of beads, previously submitted to packing in a stainless steel column, were evaluated to search for beads collapse. This would indicate the resistance of the beads under medium pressure (1345 psi) in the stainless steel column packing. No cracked beads or fragments were found in this investigation, proving that the cross-linked beads were mechanically resistant and the assessment

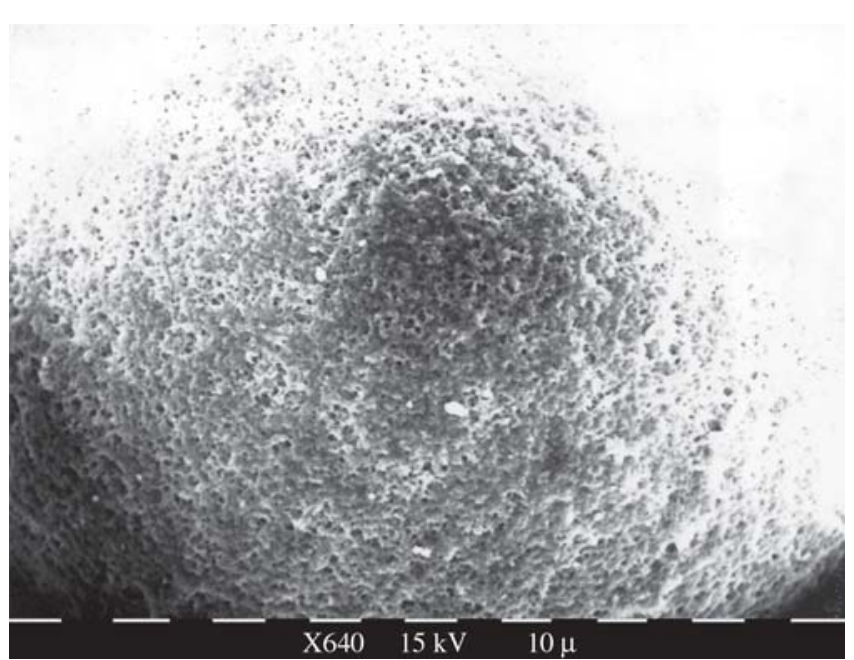

(a)

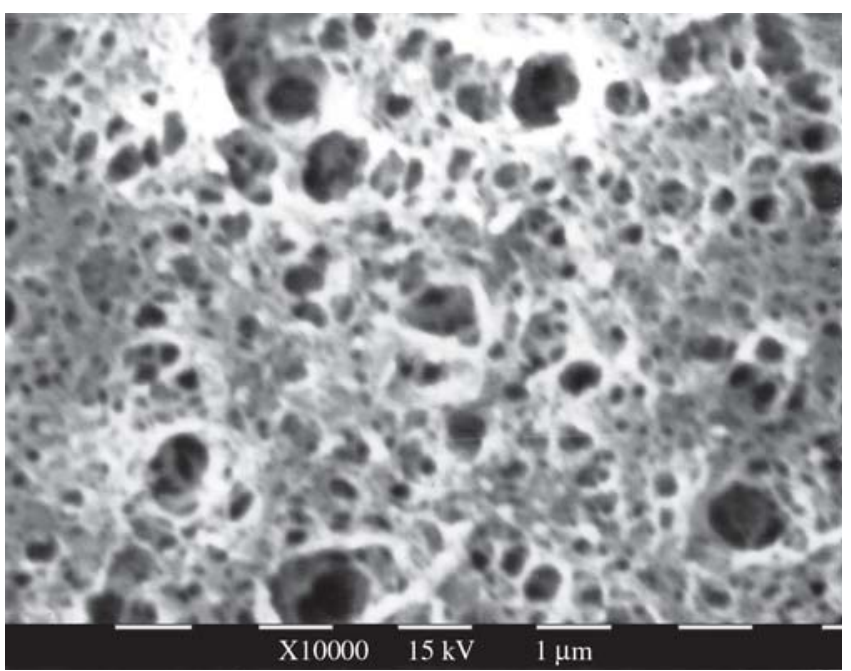

(b)

Figure 1. a) SEM photograph of copolymerized HEMA/MMA/EDMA beads, with addition of $20 \%$ porogen solvent, DOD, and 10\% EDMA after simple extraction (SE, X640, scale bar $10 \mu \mathrm{m})$; b) after Soxhlet extraction, (SOX, X10000, scale bar $1 \mu \mathrm{m})$ 
of the chromatography experiment could proceed.

In the SEC separation profile of the HEMA/MMA/EDMA packing material, it was expected that the peaks come out broad and short, due to the large pores and particle size of the packed beads. These results were confirmed by the chromatograms shown in Figures $2 \mathrm{a}$ to 2f. The overlay peaks of 6 dextran standards A, (MW 2,000,000; MW 600,000; MW 170,000; MW 75,000; MW 40,000; and MW $10,000)$, were not distinct and were superimposed over a long length of baseline. However, there appears to be a size exclusion separation process occurring in which the higher MW is eluted first out of the column and the lower MW macromolecule is the last to elute. Glucose (Figure 2g) was used as the column marker, showing total inclusion in the pores. The column can distinguish and separate very large MW compounds from low MW compounds. The HEMA/MMA/EDMA

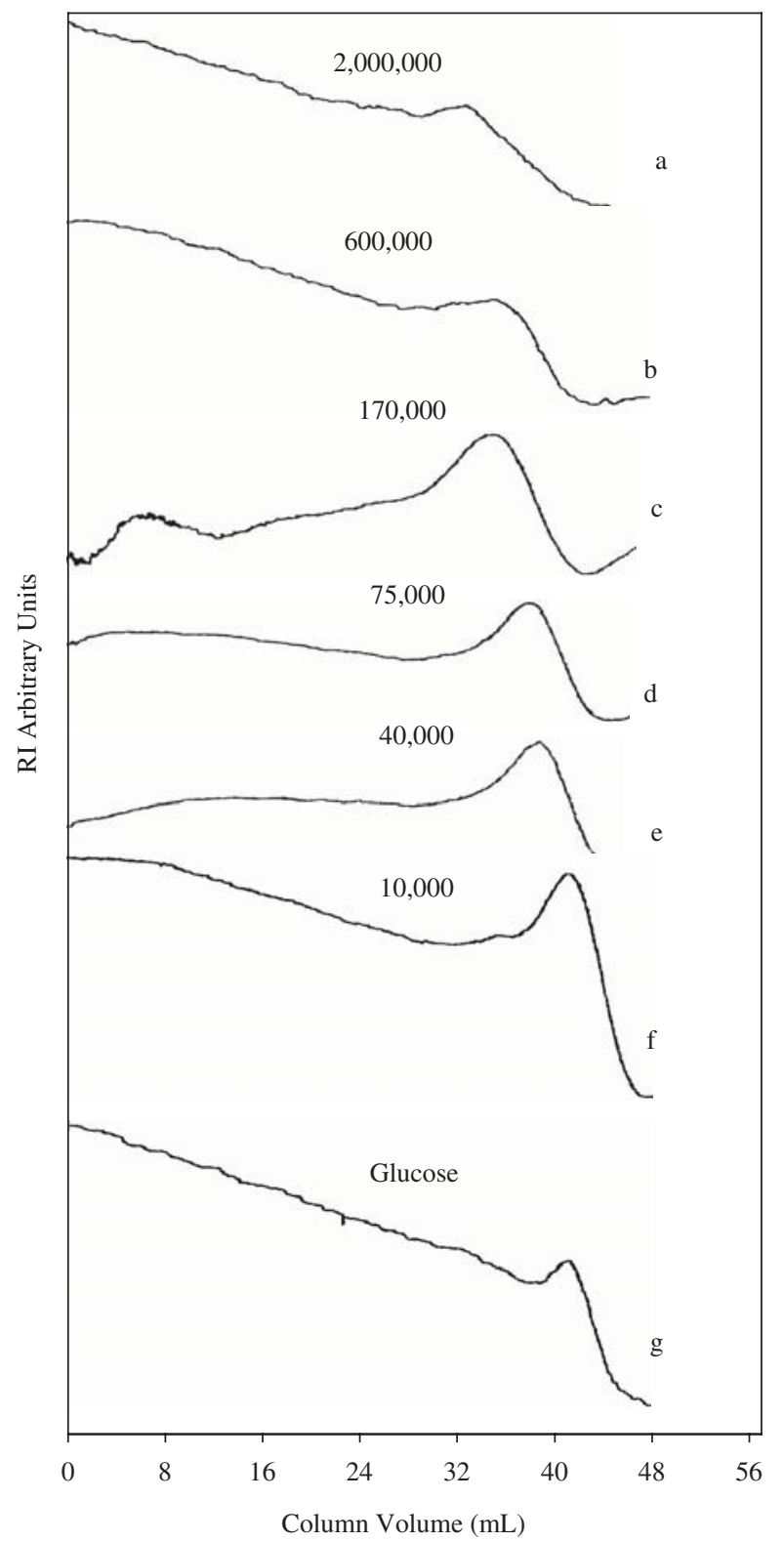

Figure 2. Representative chromatograms of Dextran standards A, a) MW 2,000,000; b) 600,000; c) 170,000 ; d) 75,000; e) 40,000; f) 10,000; g) glucose injected in the HEMA/MMA/EDMA packed glass column $(50 \mathrm{x}$ $1.5 \mathrm{~cm}$ ), using deionized distilled water as mobile phase, $1.0 \mathrm{~mL} / \mathrm{min}$ flow rate, RI detector, $100 \mu \mathrm{L}$ and $10 \mathrm{mV}$ register attenuation. material packed in the glass column can accommodate a very large quantity of sample $(100-1000 \mu \mathrm{L})$, and this feature itself is very attractive for preparative use. The HEMA/MMA/EDMA packed stainless steel column showed that the dextran separations could not be as well distinguished (Figure not shown) as they were in the glass packed columns. Even though using a lower flow rate in the stainless steel column $(0.3 \mathrm{~mL} / \mathrm{min})$ than in the glass column $(1.0 \mathrm{~mL} / \mathrm{min})$ the solute elution was not distinct because the macromolecules needed more column time and length for interaction.

The chromatographic results of the dextran standards A, (MW 2,000,000; MW 600,000; MW 170,000; MW 75,000; MW 40,000 and MW 10,000), injected in the HEMA/MMA/EDMA packed glass and steel columns, are presented in Table 1. Calibration data for dextran standards A and non-standards B are shown in Table 2. Both glass and steel columns yielded the same linear MW range (MW 2,000,000-40,000) using either one dextran series (A or B).

HEMA/MMA/EDMA copolymer calibration curve is shown in Figure 3. Figure 3a shows the linear relationship of the logarithm of dextran reference standard MW (independent variable, though represented in the $\mathrm{Y}$ axis) $v s$. the retention factor, $k$ (dependent variable, represented in the $\mathrm{X}$ axis). The linear portion of the curve is

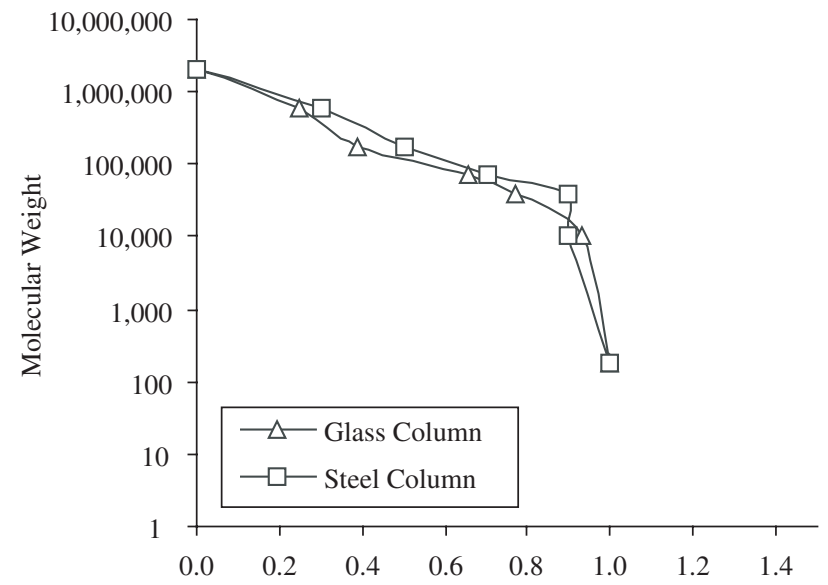

(a)

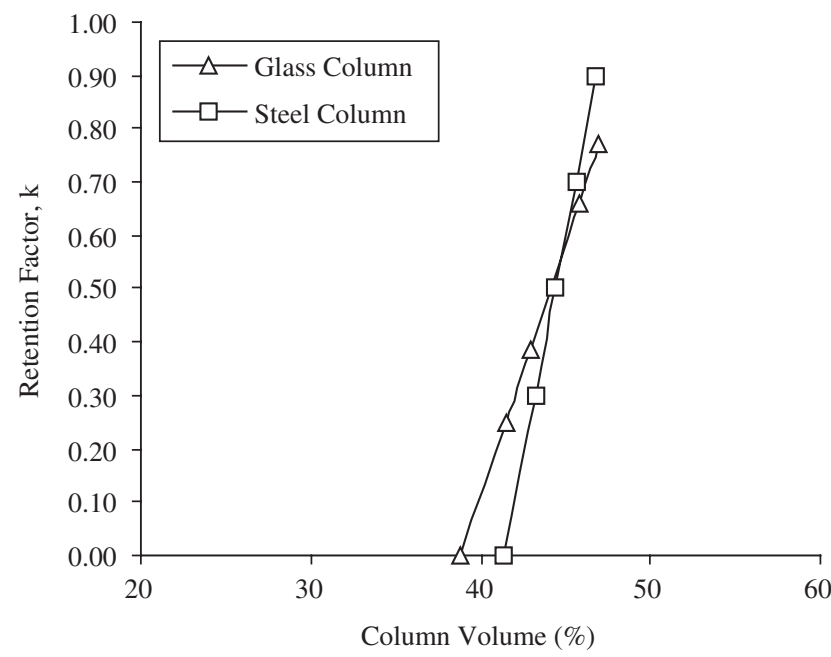

(b)

Figure 3. a) MW calibration curve of Dextran standards A in respect to retention factor, $\mathrm{k}$; b) the effective column volume separation range, of Dextran standards A injected in the HEMA/MMA/EDMA glass $(\Delta)$ and stainless steel $(\square)$ columns. 
Table 1. SEC retention parameters of dextran standards A using HEMA/MMA/EDMA packing material in the glass and stainless steel columns.

\begin{tabular}{|c|c|c|c|c|c|c|c|}
\hline Compound & \multicolumn{6}{|c|}{ Dextran Standards A } & Glucose \\
\hline Molecular Weight (MW) & $2,000,000$ & 600,000 & 170,000 & 75,000 & 40,000 & 10,000 & 180 \\
\hline $\begin{array}{l}\text { Molecular Weight Disper- } \\
\text { sity }(\mathrm{Mw} / \mathrm{Mn})^{\mathrm{b}}\end{array}$ & 11.2 & 1.5 & 2.0 & 1.6 & 1.6 & 1.5 & - \\
\hline \multicolumn{8}{|l|}{ Glass Column } \\
\hline $\begin{array}{l}\text { Retention Distance (Rd, } \\
\mathrm{cm})\end{array}$ & 8.05 & 8.60 & 8.90 & 9.50 & 9.75 & 10.10 & 10.25 \\
\hline $\begin{array}{l}\text { Retention Volume (Ve, } \\
\mathrm{mL})^{\mathrm{c}}\end{array}$ & 32.20 & 34.40 & 35.60 & 38.00 & 39.00 & 40.40 & 41.00 \\
\hline$\%$ Column Volume ${ }^{\mathrm{d}}$ & 39.00 & 41.00 & 43.00 & 46.00 & 47.00 & 49.00 & 49.00 \\
\hline Retention Factor (k) & 0 & 0.25 & 0.39 & 0.66 & 0.77 & 0.93 & 1.00 \\
\hline Separation Factor $(\alpha)$ & & 1.55 & 1.71 & 1.17 & 1.21 & 1.07 & \\
\hline $\begin{array}{l}\text { Column Volume Parameters } \\
(\mathrm{mL})\end{array}$ & Vo & 32.2 & $\mathrm{Vm}$ & 41.0 & $\mathrm{Vi}$ & 8.8 & \\
\hline \multicolumn{8}{|l|}{ Steel Column } \\
\hline Retention Distance (cm) & 1.73 & 1.80 & 1.85 & 1.90 & 1.95 & 1.95 & 1.98 \\
\hline $\begin{array}{l}\text { Retention Volume }{ }^{\mathrm{c}}(\mathrm{Ve}, \\
\mathrm{mL} \text { ) }\end{array}$ & 2.07 & 2.16 & 2.22 & 2.28 & 2.34 & 2.34 & 2.37 \\
\hline$\%$ Column Volume ${ }^{\mathrm{e}}$ & 41 & 43 & 44 & 46 & 47 & 47 & 47 \\
\hline Retention Factor (k) & 0 & 0.30 & 0.50 & 0.70 & 0.90 & 0.90 & 1.00 \\
\hline Separation Factor $(\alpha)$ & & & 1.67 & 1.40 & 1.29 & 1.00 & 1.11 \\
\hline $\begin{array}{l}\text { Column Volume Parameters } \\
(\mathrm{mL})\end{array}$ & Vo & 2.07 & $\mathrm{Vm}$ & 2.37 & $\mathrm{Vi}$ & 0.30 & \\
\hline
\end{tabular}

a: Chromatographic conditions in deionized, distilled water at room temperature, glass $(50 \times 1.5 \mathrm{~cm}, 1.5 \mathrm{~mL} / \mathrm{min}) \mathrm{and} \mathrm{steel}(300 \times 4.6 \mathrm{~mm}, 0.3 \mathrm{~mL} / \mathrm{min})$ column with RI detection; chart speed (s) $0.25 \mathrm{~cm} / \mathrm{min} ; b$ : Reported by manufacturer; $c$ : Average of two determinations; $d$ : Total column volume $83 \mathrm{~mL} ; e$ : Total column volume $5 \mathrm{~mL}$.

Table 2. Calibration curves parameters $(Y=-b \log X+a)$ for dextrans using HEMA/MMA/EDMA packed glass and stainless steel columns.

\begin{tabular}{lccc}
\multicolumn{1}{c}{ Parameters } & Slope, $\mathrm{b}(\mathrm{se})^{\mathrm{b}}$ & Intercept, $\mathrm{a}(\mathrm{se})^{\mathrm{b}}$ & Linearity, $\mathrm{L}$ \\
\hline Dextran standards A & & \\
Glass Column & $-0.2660(0.02)$ & $14.8660(0.71)$ & 0.9895 \\
Stainless Steel Column & $-6.5203(0.39)$ & $19.7933(0.85)$ & 0.9948 \\
Dextran non-standards B & & $2,000,000-40,000$ \\
Glass Column & $-0.4877(0.01)$ & $24.1719(0.56)$ & 0.9991 \\
Stainless Steel Column & $-6.8820(0.71)$ & $21.0634(1.62)$ & 0.9895 \\
\hline
\end{tabular}

$a$ : $95 \%$ confidence level; $b$ : (se): standard error; $r$ : correlation coefficient.

the effective range of separation. Figure $3 \mathrm{~b}$ demonstrates the column linear window for separation of dextran macromolecules. The effective range, in fact is very narrow, thus, this column is best for the separation of macromolecules with distinct MW.

Figure 4 shows the injection of the polysaccharides Xanthan Gum/ XAG (MW > 1,000,000), Sodium Alginic Acid/SAG (MW 120,000190,000) and Agarose/AGA (MW not defined), in the HEMA/MMA/ EDMA packed glass column $(50 \times 1.5 \mathrm{~cm})$. The peaks were broad and revealed a shoulder for both XAG and SAG injections. Size exclusion appeared to be the mechanism involved in this elution, as the larger macromolecule eluted first. The AGA polysaccharide was observed to have a relatively longer retention in the HEMA/MMA/ EDMA packed glass column using $0.1 \% \mathrm{w} / \mathrm{v}$ aqueous sodium azide as mobile phase at $1.5 \mathrm{~mL} / \mathrm{min}$. Separate injections of XAG and SAG samples, yielded two peaks for each injection. The major peaks of each polysaccharide were distinct with different retention volumes. The minor peaks exhibited retention volumes similar to the AGA peak injected in the same column. The presence of the minor peak in the XAG and SAG chromatograms may have been caused by interactions of diverse functional groups of the solute molecules and the column hydrophilic hydroxyl functional groups. The HEMA/MMA/EDMA packing material column did not show a distinctive elution profile for the polysaccharides injected in the stainless steel column (Figure 5), as well as shown for the column calibration. Chromatographic separations therefore, were not performed on this column.

Figure 6 shows the injection of the proteins BSA, OVA and CYT in the HEMA/MMA/EDMA packed glass column. Although this column provided a high theoretical plate number count for glucose, as seen in its calibration with dextrans, the same effect was not evident with the injection of commonly used proteins. The BSA and OVA proteins displayed an inverted elution order with high retention volumes in the long glass column. It is important to emphasize that the HEMA/MMA/EDMA material was expected to have some degree of hydrophobicity because it was made with MMA and EDMA, hydrophobic monomers used to control pore size and cross-link the polymer, respectively.

According to Smigol et al. ${ }^{11}$ BSA and OVA have a similar hydrophobicity relative to CYT, in which BSA shows a slightly higher value (0.24) compared to OVA (0.23). However, this difference may be too small to be imparting sorptive interactions which affect the elu- 
tion mechanism of BSA. The early elution of OVA may be attributed to other factors. For example, electrostatic forces may be involved. OVA has an isoelectric point (pI 4.6) lower than BSA (pI 5.0). In the $\mathrm{pH}$ of the mobile phase (6.5) the OVA will have a preponderance of negative charges relative to BSA resulting in repulsive interactions causing the OVA to elute faster.

Several investigators have tried to diminish the electrostatic interactions of proteins with the matrix by changing the ionic strength, the $\mathrm{pH}$ or the dielectric constant of the mobile phase. Using a polymer based column (Toyopearl ® HW55F), Germershausen and Karkas ${ }^{12}$ have found that high ionic strength may affect low MW compounds (for instance, Cytochrome C, MW 12,500) towards the reduction of retention time more than high MW compounds (e.g., thyroglobulin, MW 670,000 or aldolase, MW 158,000). In a similar study using the same matrix, Inouye ${ }^{13}$ investigated the addition of $0.3-0.5 \mathrm{M} \mathrm{NaCl}$ in the mobile phase ( $0.25 \mathrm{M}$ TRIS- $\mathrm{HCl}$ buffer, $\mathrm{pH} 7.5)$. The results showed a diminished retardation of all proteins with $\mathrm{pI}$ higher than 7.5, however, the elution order did not reflect a size exclusion mechanism. The author suggests that electrostatic forces are involved between the proteins and the gel matrix. It is worthwhile to mention that low temperatures $\left(5-10^{\circ} \mathrm{C}\right)$ were used in that study. Nevertheless, it is difficult to explain the protein elution behavior based upon electrostatic and hydrophobic forces alone ${ }^{13}$. Potschka ${ }^{14}$ and Pujar and Zydney ${ }^{15}$ state that an increase of the ionic strength would reduce the electric double layer of the protein compressing it, whilst a low ionic strength would increase the double layer, increasing the hydrodynamic size and therefore reducing the retention volume. Potschka ${ }^{14}$ however,

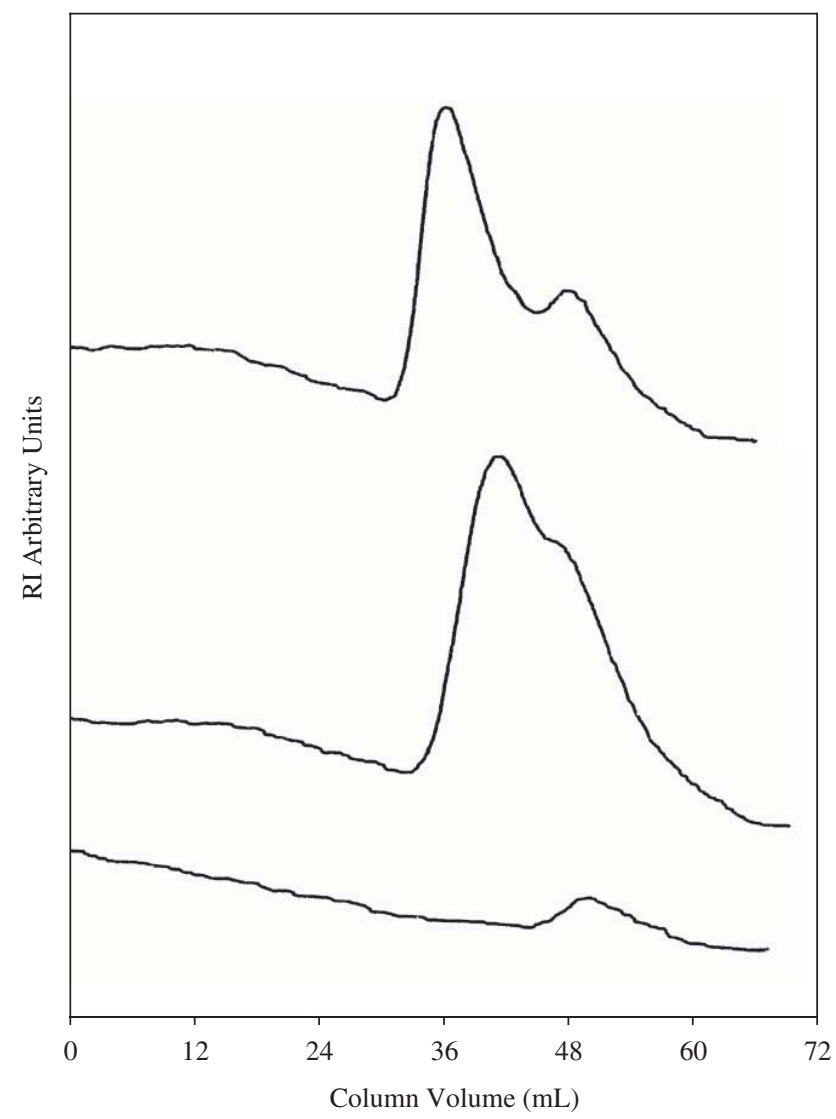

Figure 4. Representative chromatograms of polysaccharides XAG, SAG and AGA aqueous solution injected in the HEMA/MMA/EDMA packed glass columns $(50 \times 1.5 \mathrm{~cm})$, using $0.1 \% \mathrm{w} / \mathrm{v}$ aqueous sodium azide as mobile phase, $1.5 \mathrm{~mL} / \mathrm{min}$ flow rate, $\mathrm{RI}$ detector and $10 \mathrm{mV}$ register attenuation. limits the factors that govern polyelectrolyte macromolecules only to microporous matrices.

In an attempt to investigate protein behavior in the HEMA/MMA/

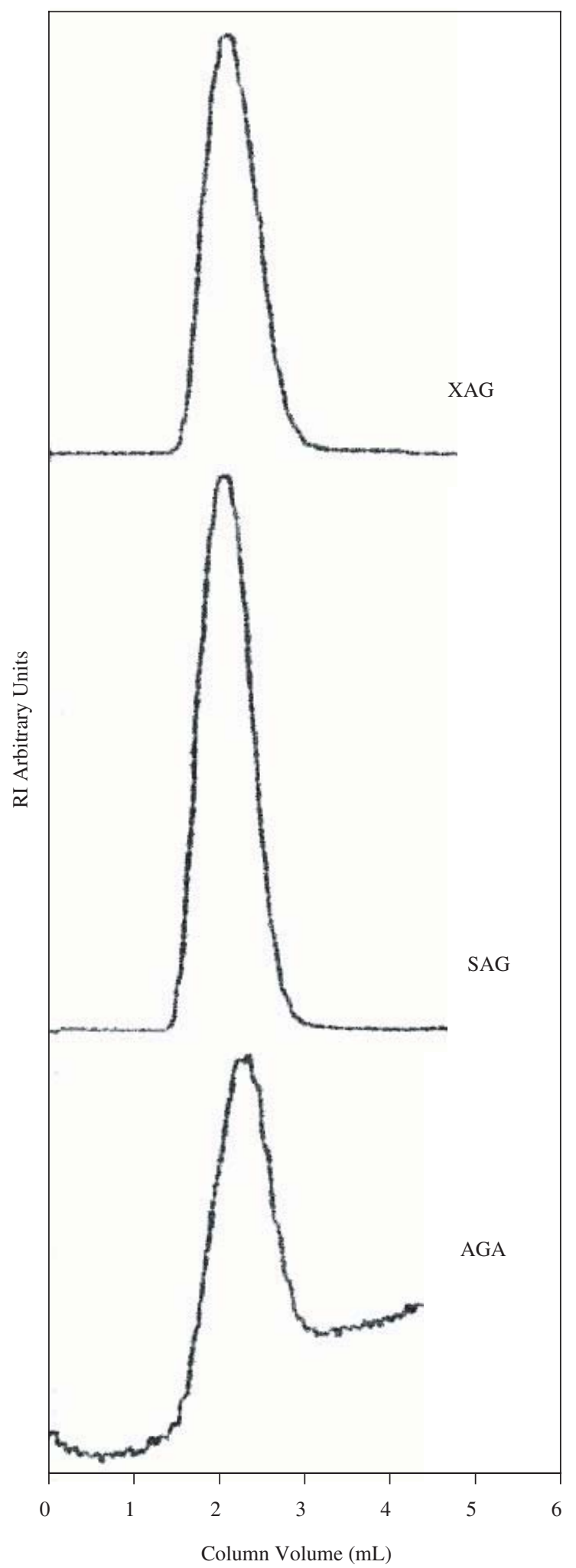

Figure 5. Representative chromatograms of polysaccharides XAG, SAG and AGA aqueous solution injected in the HEMA/MMA/EDMA packed stainless steel columns $(300 \times 4.6 \mathrm{~mm})$, using $0.1 \% \mathrm{w} / \mathrm{v}$ aqueous sodium azide as mobile phase, $0.3 \mathrm{~mL} / \mathrm{min}$ flow rate, RI detector and $100 \mathrm{mV}$ register attenuation. 


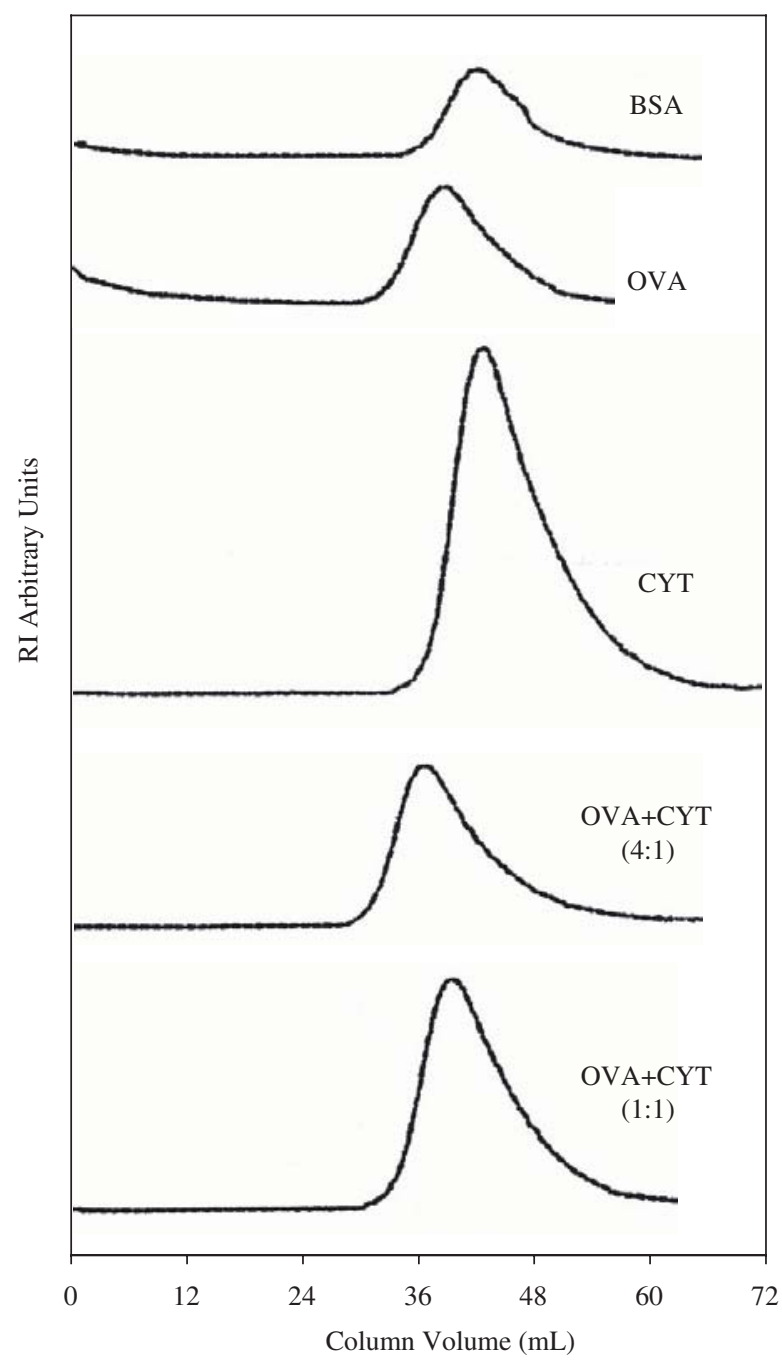

Figure 6. Representative chromatograms of polypeptides samples BSA, OVA, CYT, the 4:1 ratio OVA:CYT mixture and the 1:1 ratio OVA:CYT mixture injected in the HEMA/MMA/EDMA packed glass column $(50 \times 1.5 \mathrm{~cm})$, using $0.01 \mathrm{M}$ PBS containing $0.2 \mathrm{M}$ sodium sulfate, $\mathrm{pH} 6.5$ as mobile phase, $1.5 \mathrm{~mL} /$ min flow rate, $\mathrm{UV}(280 \mathrm{~nm})$ detector and $100 \mathrm{mV}$ register attenuation.

EDMA column, the ionic strength and $\mathrm{pH}$ of the mobile phase were modified. In the mobile phase $0.01 \mathrm{M}$ PBS pH 6.5 containing sodium sulfate, high (0.2 M, Figure 6) and low (0.002 M, Figure not shown) ionic strength were tested. The results using low ionic strength indicated no change in the elution profile of BSA and OVA when compared to the phase with high ionic strength. In fact, not only the elution order of BSA/OVA was inverted, but also CYT was much retarded as in the low ionic strength $(0.02 \mathrm{M}) 0.01 \mathrm{M}$ PBS pH 6.5 phase. This non-ideal behavior due to interaction with the support must be eliminated in order to estimate $\mathrm{MW}^{16}$.

The proteins in a $\mathrm{pH} 8.0$ mobile phase were then tested with $0.01 \mathrm{M}$ TRIS- $\mathrm{HCl}$ containing a low concentration of sodium sulfate $(0.002 \mathrm{M})$. BSA and OVA elution order was not inverted. Actually, both proteins presented the same elution volume (Figure 7). This confirms that electrostatic repulsive forces were the major cause of the inverted elution profile in the $0.01 \mathrm{M}$ PBS pH 6.5 mobile phase. On the other hand, the large size of pores in HEMA/MMA/EDMA may very well be the major determinant of the non-adherence to a size exclusion mechanism for these polypeptides (lower MW) compared to the polypeptides of higher MW.

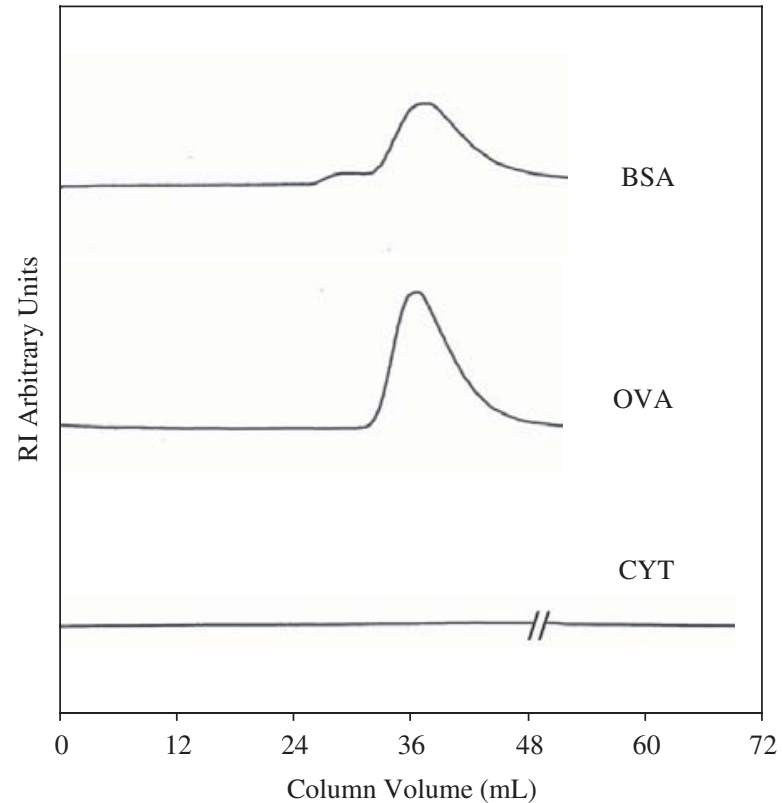

Figure 7. Representative chromatograms of polypeptides samples BSA, OVA, and CYT injected in the HEMA/MMA/EDMA packed glass column $(50 \times 1.5 \mathrm{~cm})$, using $0.01 \mathrm{M}$ TRIS- $\mathrm{HCl}$ containing $0.002 \mathrm{M}$ sodium sulfate, $\mathrm{pH} 8.0$ as mobile phase, $1.5 \mathrm{~mL} / \mathrm{min}$ flow rate, $\mathrm{UV}(280 \mathrm{~nm})$ detector and $10 \mathrm{mV}$ register attenuation.

\section{Conclusion}

The separation profile of macromolecules was demonstrated by the use of a hydrophilic synthetic packing material (copolymer HEMA/MMA/EDMA). Polysaccharides samples of high molecular weight can be separated by the size exclusion mechanism with the use of an appropriate mobile phase. Further studies to clarify and demonstrate the potential of the developed material for the separation of polypeptides of much higher molecular weights have been carried on.

\section{Acknowledgements}

Vianna-Soares, C.D. is grateful for the financial support from Temple University and the fellowship from CAPES/Brasília/Brazil.

\section{References}

1. Moore JC. Gel Permeation Chromatography. I. A new method for molecular weight distribution of high polymers. J Pol Sci A, 1964; 2:835-843.

2. Schott, H. Polymer science. In: Martin A. Physical pharmacy. Philadelphia: Lea \& Febiger; 1993. p. 556-594.

3. Tanaka N, Kimata K, Mikawa Y, Hosoya K, Araki T, Ohtsu Y et al. Performance of wide-pore silica- and polymer-based packing materials in polypeptide separation: effects of pore size and alkyl chains length. $J$ Chromatogr. 1990; 535:13-31.

4. Reinholdsson P, Hargitai T, Isaksson R, Tornell B. Preparation and properties of porous particles form trimethylolpropane trimethacrylate. Angew Makromol Chemie. 1991; 192:113-132.

5. Barth HG, Boyes BE, Jackson C. Size exclusion chromatography. Anal Chem.1994; 66:595R-620R.

6. Mendichi R, Rizzo V, Gigli M, Razzano G. Molar mass distribution of a polymer-drug conjugate containing the antitumor drug paclitaxel by size exclusion chromatography and universal calibration. J Liq Rel Technol. 1998; 21(9):1295-1309.

7. Coupek J, Vins I. Hydroxyethyl methacrylate-based sorbents for highperformance liquid chromatography of proteins. J Chromatogr A. 1994; 658:391-398. 
8. Vianna-Soares CD, Kim CJ, Borenstein MR. Manufacture of porous cross-linked HEMA spheres for size exclusion packing material. J Porous Mater. 2003; 10:123-130.

9. Leon y Leon CA, Thomas MA. Recent advances in the interpretation of mercury porosimetry data. G.I.T. Lab J Int Ed. 1997;1:101-104.

10. Lowell S, Shields JE. Powder surface area and porosity. London: Chapman \& Hall, 1991

11. Smigol V, Svec F, Frechet J.M.J. Two-dimensional high-performance liquid chromatography using monodisperse polymer beads containing segregated chemistries prepared by pore size specific functionalization. Single-column combinations of size exclusion or ion exchange with reversed-phase chromatography. Anal Chem. 1994; 66:2129-2138.

12. Germershausen J, Karkas JD. Preparative high speed gel permeation chromatography of proteins on Toyopearl HW55F. Biochem Biophys Res Comm, 1981; 99(3):1020-1027.

13. Inouye $\mathrm{K}$. Chromatographic behaviors of proteins and amino acids on a gel filtration matrix, TSK-GEL Toyopearl. Agric Biol Chem, 1991; 55(8):2129-2139.

14. Potschka M. Size-exclusion chromatography of polyelectrolytes experimental evidence for a general mechanism. J Chromatogr, 1988; 441:239260.

15. Pujar NS, Zydney AL. Electrostatic effects on protein partitioning in sizeexclusion chromatography and membrane ultrafiltration. J Chromatogr A, 1998; 796:229-238.

16. Cunico RL, Gooding KM. Wehr T. Basic HPLC and CE of biomolecules. Bioanalytical Laboratory, Richmond: Bioanalytical Laboratory; 1998. p.123-144. 